\title{
Special issue on machine learning algorithms for internet of things, fog computing and cloud computing
}

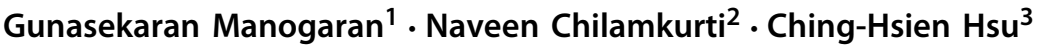

Published online: 10 July 2018

(c) Springer-Verlag GmbH Austria, part of Springer Nature 2018

Nowadays, IoT devices such as wearable medical devices, smart traffic control devices, and various IoT sensors are continuously generated a massive amount of data. This huge amount of data is not possible to process by traditional tools and technologies. Hence, there is a need for scalable machine learning algorithms to process such massive amount of data. In recent years, a number scalable machine learning algorithms are developed to process the massive IoT data. This special issue focused on innovations in scalable machine learning algorithms, novel IoT architecture, integration of fog and cloud computing, and processing of big data. In addition, this issue also paying an attention to social network analysis, and security and privacy in Internet of Things, Fog Computing and Cloud Computing.

The first paper, entitled A Novel Machine Learning Framework for Diagnosing the Type 2 Diabetics using Temporal Fuzzy Ant Miner Decision Tree Classifier with Temporal Weighted Genetic Algorithm, by Bhuvaneswari and Manikandan, proposed the new temporal weighted genetic algorithm for enhancing the detection accuracy by preprocessing the text and image data. Authors have empirically evaluated the effectiveness of the proposed TFAMT-TWGA model using the UCI Repository dataset and the collected retinopathy image dataset.

The second paper, entitled PTL-CFS based Deep Convolutional Neural Network Model for Remote Sensing Classification, by Xiaodong Yu and Hongbin Dong, proposed a Convolutional Neural Network Remote Sensing Classification Model based

Gunasekaran Manogaran

gunavit@gmail.com

Naveen Chilamkurti

n.chilamkurti@latrobe.edu.au

Ching-Hsien Hsu

chh@chu.edu.tw

1 School of Information Technology \& Engineering, VIT University, Vellore, India

2 Department of Computer Science and Computer Engineering, LaTrobe University, Melbourne, Australia

3 Department of Computer Science and Information Engineering, Chung Hua University, Hsinchu, Taiwan 
on PTL-CFS. This model utilizes parameter transfer learning algorithm to obtain the $\mathrm{CNN}$ initialization parameters of the target area, then it uses correlation-based feature selection algorithm to eliminate the redundant features and noises from the original feature set, finally, it classifies the remote sensing images using a conventional CNN model.

The third paper, entitled Test case selection using multi-criteria optimization for effective fault localization, by Wang et. al, proposed an approach for selecting a subset of the passed test suite when a failure revealed by a failed test case. The goal is to obtain a more effective fault localization using a minimal number of test cases than using the originally given large number of test cases.

The fourth paper, entitled A Novel Recommendation System Based on Semantics and Context Awareness, by Qin Yang, proposed a two state-of-the-art methods are analyzed and extended to enhance recommendation performance. The first method is the context-aware recommender, which integrates context information into the recommendation process. The second method is the semantic analysis-based recommender, which incorporates domain semantics. Despite their compatibility, the challenge is to combine them in a way that will fully exploit their potential.

The fifth paper, entitled Prediction Algorithm of PM2.5 Mass Concentration Based on adaptive BP Neural Network, by Chen Yegang, used the BP neural network algorithm to predict the quality of PM2.5 and air quality, which indicates that it is feasible to use neural network to predict quality of the air.

The sixth paper, entitled Computing Efficient Features using Rough Set Theory combined with Ensemble Classification techniques to improve the Customer Churn Prediction in Telecommunication Sector, by Vijaya and Sivasankar, proposed the Rough Set Theory (RST) based model to identify the efficient features for telecommunication customer churn prediction. In this work the duke university-churn prediction data set is considered for performance evaluation and three sets of experiments are performed.

The seventh paper, entitled A Cooperative Particle Swarm Optimization with Constriction Factor Based on Simulated Annealing, by Zhuang Wu et. al, brought the Metropolis criterion of simulated annealing algorithm into the particle swarm optimization with constriction factor. It analyzes the impact the parameters have on the algorithm performance and the convergence, efficiency and parameter selection in different forms.

The eighth paper, entitled The Dynamic Investment Strategy of Online Advertising Based on Spillover Effect in Duopoly Competition Market, by Huini Zhou et. al, proposed the investment cost function which reflects the characteristics and impacts of online advertising spillover effect to enterprises. Then the improved Lanchester model is used based on the investment cost function.

We would like to convey our sincere thanks to all the researchers for submitting their manuscripts and a special note of thanks to the reviewers, whose efforts have allowed the selection of good quality papers. We are also grateful to the Computing Journal, for allowing us to divulge a selected sample of the ongoing research efforts on recent advancements in machine learning algorithms especially in Internet of Things, Fog Computing and Cloud Computing. 